\title{
Traffic composition and variability of road traffic noise levels in the vicinity of Colombo, Sri Lanka
}

\author{
C.M. Kalansuriya ${ }^{1}$, A.S. Pannila ${ }^{1}$ and D.U.J. Sonnadara ${ }^{2^{*}}$ \\ ${ }^{\prime}$ Electro Technology Centre, Industrial Technology Institute, 363, Bauddhaloka Mawatha, Colombo 07. \\ ${ }^{2}$ Department of Physics, Faculty of Science, University of Colombo, Colombo 03.
}

Revised: 09 September 2014; Accepted: 21 November 2014

\begin{abstract}
A study was conducted to determine the contribution of different types of vehicles to road traffic noise levels. Measurements were carried out on three different categories of roads at six separate locations, approximately $30 \mathrm{~km}$ away from the city of Colombo. At each location, continuous noise measurements were taken for a duration of 13 hours on weekdays and several key noise level descriptors $\left(\mathrm{L}_{\mathrm{eq}}, \mathrm{L}_{10}\right.$, $\mathrm{L}_{50}, \mathrm{~L}_{90}$ ) were measured simultaneously. It was observed that A-weighted equivalent noise level $\left(\mathrm{L}_{\mathrm{eq}}\right)$ along main roads and secondary roads reached $\sim 70 \mathrm{~dB}(\mathrm{~A})$, which is the maximum permissible noise level prescribed for road traffic by Japan for residential areas. Similar to previous studies, a linear correlation was observed between $\mathrm{L}_{\mathrm{eq}}$ and other noise descriptors. Specially $\mathrm{L}_{10}$, which can be related to annoyance, can be estimated with an accuracy of $\pm 1 \mathrm{~dB}(\mathrm{~A})$ by measuring $\mathrm{L}_{\mathrm{eq}}$. The correlation between the rate of vehicle flow and the measured noise level showed a logarithmic relationship. It is shown that the key noise descriptors can be estimated with an accuracy of \pm 2.5 $\mathrm{dB}(\mathrm{A})$ by measuring the flow rate of vehicles. The vehicle composition showed that heavy vehicles such as lorries, buses and containers contribute significantly to the average noise levels.
\end{abstract}

Keywords: Noise level, road traffic noise, traffic composition, vehicle noise.

\section{INTRODUCTION}

Community noise (also called environmental noise, residential noise, or domestic noise) includes noise emitted from all sources except that emitted from workplaces. The main sources of community noise in Sri Lanka are vehicular traffic, construction activities and public address systems, all of which affect in various degrees to those who live in close proximity to traffic routes. Constant exposure to noise can affect both mental and physical health. Noise can interfere with communication, cause cardiovascular effects, sleep disturbances, reduce performance, lead to annoyance and alter social behaviour. At sufficiently high levels, it can also impair hearing (Langdon, 1976; WHO, 1999; Ouis, 2001).

There are several studies related to the assessment of road traffic noise levels in different cities around the world (Onuu, 2000; Morillas et al., 2002; Ali \& Tamura, 2003; Sommerhoff et al., 2004; Piccolo et al., 2005). A study carried out to investigate road traffic noise in several cities in South-Eastern Nigeria (Onuu, 2000) has reported noise levels, which are higher than in cities in developed countries. The measured $\mathrm{L}_{10}$ values inside schools and hospitals were as high as 81 and $72 \mathrm{~dB}(\mathrm{~A})$, respectively, which is higher than the level required for communication. They have found an apparent income bias in response to annoyance caused by road traffic noise, with low income neighbourhoods reporting less disturbances and some evidence of adaptation to road traffic noise.

A study conducted in Caceres in Spain (Morillas et al., 2002) has shown that city noise can be evaluated by classifying streets according to their use. The study has shown that even for smaller non-industrial cities, traffic noise could be a major pollutant; about $90 \%$ of the measurements exceeding the $65 \mathrm{~dB}(\mathrm{~A})$ level. The results also indicated that there is a clear relationship between the road traffic noise and traffic volume as shown in a number of previous studies (Manoel et al., 2004; Piccolo et al., 2005). An acceptable linear correlation was observed between $\mathrm{L}_{\text {eq }}$ and $\mathrm{L}_{10}$ although it was not very well established for other noise descriptors. 
A study conducted in Valdivia in Spain (Sommerhoff et al., 2004) has shown that the measured noise levels in the city are comparable to those reported in literature for cities without mitigation strategies. They conclude that road traffic is the main contributor to city noise. A study in Messina in Italy (Piccolo et al., 2005) has shown that the main roads in the city are overloaded with vehicular traffic and the city noise levels exceed the Italian noise standards by roughly $10 \mathrm{~dB}(\mathrm{~A})$. They have also reported that heavy traffic is the main source of noise pollution.

A study carried out in Greater Cairo, Egypt (Ali \& Tamura, 2003) to mitigate the road traffic noise levels and improve environmental conditions has shown that with restrictions on traffic composition, town planners can improve the city environment. With restrictions on buses and commercial vehicles they have achieved a $10 \mathrm{~dB}(\mathrm{~A})$ reduction of noise levels. The effect of traffic composition on the noise generated on Brazilian roads (Manoel et al., 2004) has shown that common noise level descriptors such as $\mathrm{L}_{\text {eq }}$ and $\mathrm{L}_{10}$ can be estimated using the traffic composition.

Exposure to traffic noise is often higher in the developing countries due to improper planning and poor enforcement of regulations. Thus, action to limit and control exposure to road traffic noise is essential. Road traffic noise pollution is also severe in the cities of Sri Lanka. Preliminary data collected along the main roads were found to have the equivalent of sound pressure levels exceeding $65 \mathrm{~dB}(\mathrm{~A})$ (Sooriyaarachchi \& Sonnadara, 2008; Wewalwala \& Sonnadara, 2011). However, no systematic survey has been conducted to determine the noise levels due to traffic in different road categories in Sri Lanka.

The main objective of this study was to determine the current noise levels in roads in the vicinity of Colombo, the capital city of Sri Lanka and relate it to the volume of traffic. Traffic noise level variations during weekdays were measured on selected road segments relative to the vehicle composition. Since Sri Lanka is yet to develop standards for road traffic noise levels, the results were compared with noise levels set by Japanese noise control regulations developed in 1970 and revised in 2000 (I-INCE, 2009).

\section{METHODOLOGY}

The present study covered the noise levels of roads in the outskirts of Colombo, which have similar characteristics. Measurements were carried out on selected straight road segments situated approximately $30 \mathrm{~km}$ away from the city of Colombo, relatively free of reflections due to buildings and walls. At this distance congestion due to traffic is less and the flow of traffic is smooth. A total of 6 separate locations, each with different traffic conditions were selected for this study. The selected locations were related to three categories of roads (main roads - category A, secondary roads - category B and local roads - category C). Noise measurements were carried out approximately $5 \mathrm{~m}$ from the edge of the traffic routes and $1.5 \mathrm{~m}$ above the ground level. At each location, noise measurements were taken for a duration of $13 \mathrm{~h}$ on weekdays. Several noise level descriptors $\left(\mathrm{L}_{\text {eq }}, \mathrm{L}_{10}, \mathrm{~L}_{50}, \mathrm{~L}_{90}, \mathrm{~L}_{\text {max }}\right.$ and $\mathrm{L}_{\text {min }}$ ) were recorded during the noise level survey. The sampling time interval was kept at $10 \mathrm{~min}$ to allow continuous recording of noise levels. The approximate number of vehicles per hour, categorized as heavy (buses, containers, lorries and bowsers), light (cars, vans, jeeps and cabs) and very light (three-wheelers and motor bikes) were also recorded simultaneously.

Noise levels were measured using the Noise Level Analyzer, BZ 2260 Version 2 Bruel \& Kjaer (class-1). The noise level meter was calibrated before and after taking the measurements each day using the noise level calibrator, B \& K Type 4230, which is traceable to the primary standards maintained at the Korea Research Institute of Standards and Science (KRISS). All noise level data were saved during measurement and the data were analysed offline by the enhanced sound analysis software, Bural \& Kajer BZ 7202, which conforms to the International Electrotechnical Commission (IEC) specific standard.

\section{RESULTS AND DISCUSSION}

\section{Traffic volume}

The variation of traffic volume (vehicles per minute) with the time of the day is shown in Figure 1(a). In general, a high traffic volume was observed during the morning and evening sessions, compared to the afternoon and night sessions in category A and category $B$ roads. Mornings and evenings are typical rush hours in Sri Lanka with a large number of office, school and public transport vehicles transporting passengers through main traffic routes. In addition, very light vehicles such as three-wheelers and motor bikes add to the traffic during these times. Without considering the vehicle type, the average traffic volume in category $\mathrm{B}$ and category $\mathrm{C}$ roads compared to the traffic volume in category A roads is 60 and $14 \%$, respectively. 


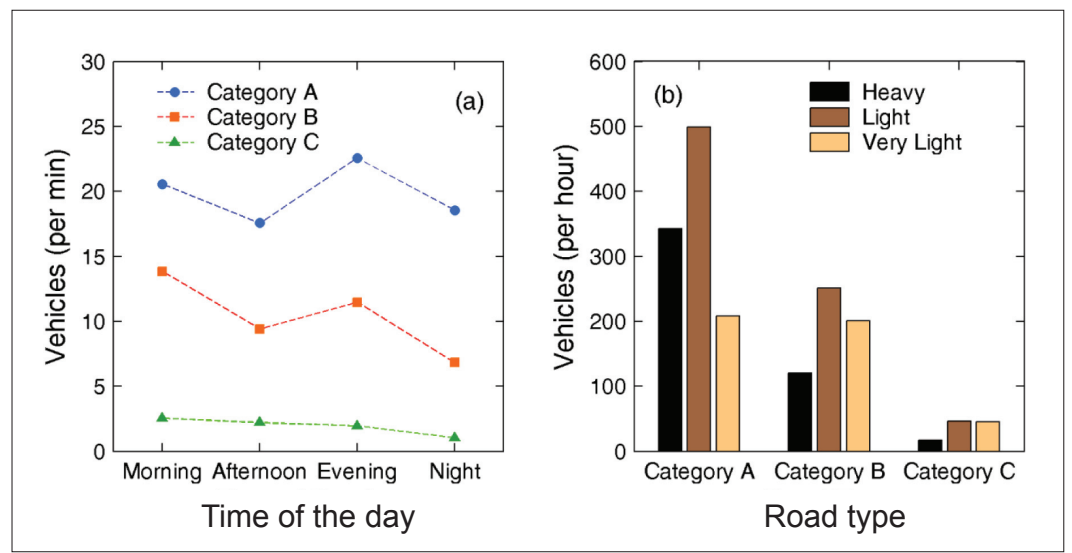

Figure 1: (a) Variation of traffic volume with time of the day for four different time segments for category A, B and C roads; (b) vehicle composition for different road types

Figure 1(b) shows the traffic volumes for different types of roads. The traffic was categorized as heavy, light and very light. For category A roads, a high level of traffic was observed for all types of vehicles. Compared to category A roads, heavy and light vehicles in category B roads were much less ( $\sim 30$ and $50 \%$, respectively) but the number of very light vehicles was similar. As expected in category $\mathrm{C}$ roads, traffic volumes were much less for all vehicle classes (compared to category A roads, approximately $5 \%$ of heavy vehicles, $10 \%$ light vehicles and $30 \%$ of very light vehicles).

\section{Noise level variation}

The noise level data based on the road category are shown in Table 1 for several noise level descriptors. If we consider $\mathrm{L}_{90}$ to be the level close to the background noise levels, the data shows that the equivalent average noise levels are significantly high in all three road categories. On average, category A, B and C roads show somewhat similar enhancement of equivalent noise levels exceeding
$15 \mathrm{~dB}(\mathrm{~A})$ due to traffic, compared to the background noise levels. Since the measurement locations for category A and $\mathrm{B}$ roads can be considered as medium noise areas and category $\mathrm{C}$ roads as low noise areas, noise exposure within business premises and dwellings located close to all the categories of roads experience more than the acceptable limits for maximum permissible levels for medium and low noise areas. The data shows that $\mathrm{L}_{10}$ exceeds day time maximum permissible noise levels by approximately $10 \mathrm{~dB}(\mathrm{~A})$ for all road categories. If one considers $\mathrm{L}_{10}$ to be correlated with the annoyance felt by people living close to traffic routes, then on average, a reduction of $10 \mathrm{~dB}(\mathrm{~A})$ is required in all three categories of roads.

The overall equivalent mean noise value $\left(\mathrm{L}_{\mathrm{eq}}\right)$ for category $\mathrm{A}, \mathrm{B}$ and $\mathrm{C}$ roads are 74,68 and $61 \mathrm{~dB}(\mathrm{~A})$, respectively. When night-time noise measurements were included, a slightly higher variation of noise levels was observed due to the reduction of vehicle flow rate during night time.

Table 1: Summary of the measured noise levels during day time $(7.00 \mathrm{am}-8.00 \mathrm{pm})$

\begin{tabular}{cccccccc}
\hline $\begin{array}{c}\text { Road } \\
\text { category }\end{array}$ & $\begin{array}{c}\text { Traffic rate } \\
\text { (per min) }\end{array}$ & $\mathrm{L}_{\text {eq }}$ & $\mathrm{L}_{90}$ & $\mathrm{~L}_{50}$ & $\mathrm{~L}_{10}$ & $\mathrm{~L}_{\text {max }}$ & $\mathrm{L}_{\text {min }}$ \\
\hline $\mathrm{A}_{1}$ & 20.2 & $74+2$ & $58+2$ & $68+2$ & $76+1$ & 109 & 44 \\
$\mathrm{~B}_{2}$ & 11.6 & $68+2$ & $53+2$ & $62+2$ & $71+2$ & 101 & 37 \\
$\mathrm{C}_{2}$ & 2.2 & $61+2$ & $44+4$ & $51+4$ & $63+2$ & 95 & 37 \\
\hline
\end{tabular}

\footnotetext{
${ }^{1}$ Two way, double lane

${ }^{2}$ Two way, single lane
} 
The noise level difference among the three categories of roads can be depicted by calculating the frequency distributions of the measured $\mathrm{L}_{\mathrm{eq}}$ value at $1 \mathrm{~dB}(\mathrm{~A})$ intervals as shown in Figure 2(a). It can be observed that the measured data symmetrically spreads around the mean value and can be reasonably fitted by Gaussian distributions with squared correlation coefficients of 0.97 , 0.78 and 0.96 for category A, B and C roads, respectively. The fitted mean values and standard deviations are; $74+1,68+1$ and $60+1 \mathrm{~dB}(\mathrm{~A})$ for category A, B and C roads, respectively.

The correlation between $\mathrm{L}_{\mathrm{eq}}$ and the percentile levels $\mathrm{L}_{10}$ and $\mathrm{L}_{90}$ are shown in Figure 2(b). Similar to the findings in a previous work (Ali \& Tamura, 2003), a linear relationship between $\mathrm{L}_{\mathrm{eq}}$ and other noise descriptors was observed. For $\mathrm{L}_{10}$, measurements can be fitted by the linear relationship $L_{10}=0.97 \times L_{e q}+4.62$ with a correlation coefficient of $\mathrm{R}^{2}=0.96$. The relationship between $\mathrm{L}_{\mathrm{eq}}$ and $\mathrm{L}_{90}$ is; $L_{90}=1.00 \times L_{e q}-15.61$ with a correlation coefficient of $\mathrm{R}^{2}=0.86$. The correlation between $\mathrm{L}_{\mathrm{eq}}$ and $\mathrm{L}_{10}$ is much stronger than the correlation between $\mathrm{L}_{e q}$ and $\mathrm{L}_{90}$. The results suggest that reliable prediction of $\mathrm{L}_{10}$ and $\mathrm{L}_{90}$ can be made once $\mathrm{L}_{\mathrm{eq}}$ is known with accuracies of approximately $\pm 1 \mathrm{~dB}(\mathrm{~A})$ and $\pm 2 \mathrm{~dB}(\mathrm{~A})$, respectively.

Figure 3(a) shows the correlation between percentile levels $\mathrm{L}_{10}, \mathrm{~L}_{50}$ and $\mathrm{L}_{90}$ against the vehicle flow rate. It was found that the general trend can be

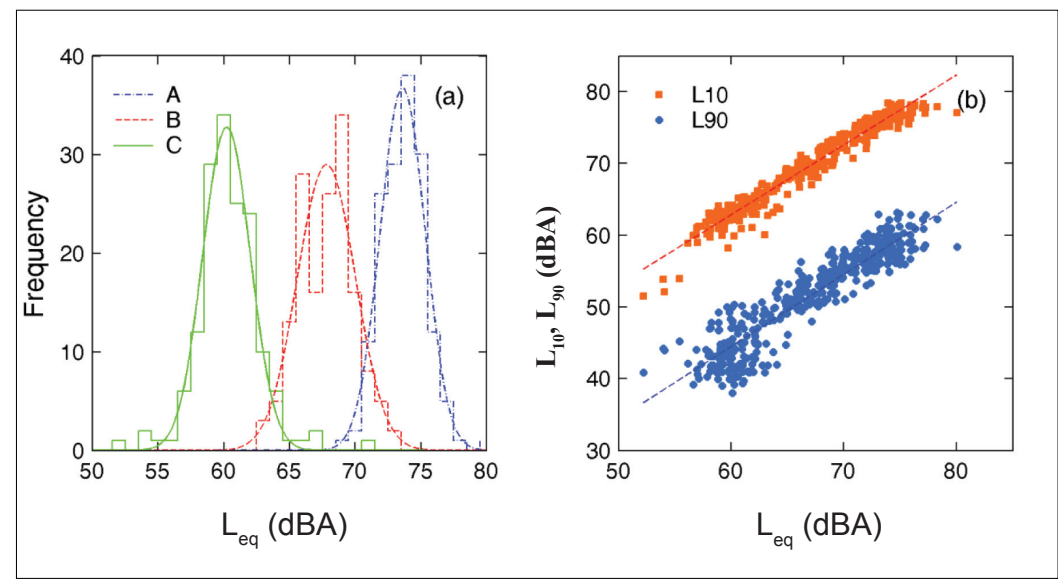

Figure 2: (a) Difference in traffic noise levels $\mathrm{L}_{\mathrm{eq}}$ between the three categories of roads. Smooth curves represent the Gaussian fit of the data points; (b) relationship between $\mathrm{L}_{\mathrm{eq}}$ and $\mathrm{L}_{10}$ and $\mathrm{L}_{90}$. Dashed lines represent the linear fit to the data.

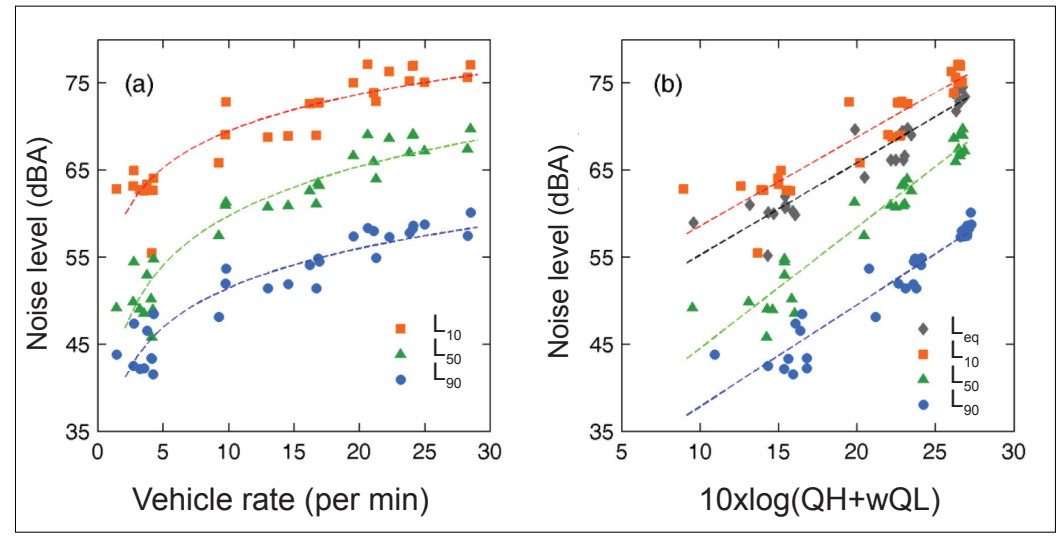

Figure 3: (a) Relationship between the measured percentile levels $\mathrm{L}_{10}, \mathrm{~L}_{50}, \mathrm{~L}_{90}$ and vehicle flow rate. The dashed lines represent the logarithmic fit to the data points; (b) $\mathrm{L}_{\mathrm{eq}}$, percentile levels $\mathrm{L}_{10}, \mathrm{~L}_{50}, \mathrm{~L}_{90}$ as a function of the logarithm of traffic composition. The value of $w$ which varies between 0.5 and 1.1 was found by minimizing the $\chi^{2}$. 
expressed using a logarithmic relationship. For $\mathrm{L}_{50}$, the noise level can be described by the relationship $L_{50}=8.16 \times \operatorname{In}(x)+40.98$ with a correlation coefficient of $\mathrm{R}^{2}=0.88$ where $x$ is the vehicle rate per minute. The relationship for the remaining noise level descriptors are; $L_{10}=6.07 \times \operatorname{In}(x)+55.70$ with a correlation coefficient of $\mathrm{R}^{2}=0.78$, and $L_{90}=6.53 \times \operatorname{In}(x)+36.47$ with a correlation coefficient of $\mathrm{R}^{2}=0.87$. This result suggests that the estimation of $\mathrm{L}_{10}, \mathrm{~L}_{50}$ and $\mathrm{L}_{90}$ can be made once the vehicle flow rate is known. When the standard deviation of the differences between the predicted (through the logarithmic relationship) and measured noise levels were calculated, it was observed that $\mathrm{L}_{10}, \mathrm{~L}_{50}$ and $\mathrm{L}_{90}$ can be predicted with accuracies of approximately $\pm 2.5 \mathrm{~dB}(\mathrm{~A})$.

\section{Vehicle composition}

In order to find the mathematical relationship between road noise and traffic composition, the following expression was used based on two previous studies (Ali \& Tamura, 2003; Manoel et al., 2004).

$$
L_{x}=a+b \times \log _{10}\left(Q_{H}+w \times Q_{L}\right)
$$

Here, $L_{x}$ refers to the average noise level of the selected noise descriptor, $a$ and $b$ are linear regression coefficients, $Q_{H}$ and $Q_{L}$ are the number of vehicles per hour for heavy and light vehicles respectively, and $w$ is a weight factor that controls the contribution of light vehicles to the overall noise level. It should be noted that $w$ will vary between 0 (no contribution from light vehicles) and 1 (all vehicles will contribute equally).

The unknown coefficients $a, b$ and $w$ can be found by fitting a straight line to the data. When minimizing $\chi^{2}$, the weight factor was allowed to vary from 0 to 1 in steps of 0.001 and for each step, the parameters $a$ and $b$, which produced the best agreement with the measurements were extracted. Based on the results, the following expressions were found to predict the noise levels for the selected noise descriptors.

$L_{e q}=44.65+10.60 \times \log _{10}\left(Q_{H}+0.06 \times Q_{L}\right) \mathrm{dB}(\mathrm{A})$ with $\mathrm{r}^{2}=0.89$

$L_{10}=48.34+10.23 \times \log _{10}\left(Q_{H}+0.05 \times Q_{L}\right) \mathrm{dB}(\mathrm{A})$ with $\mathrm{r}^{2}=0.85$

$L_{50}=30.60+13.92 \times \log _{10}\left(Q_{H}+0.06 \times Q_{L}\right) \mathrm{dB}(\mathrm{A})$ with $\mathrm{r}^{2}=0.92$

$L_{90}=26.12+11.71 \times \log _{10}\left(Q_{H}+0.11 \times Q_{L}\right) \mathrm{dB}(\mathrm{A})$ with $\mathrm{r}^{2}=0.90$

The results show that the contribution of light vehicles on the overall noise level is small $(w \approx 0.05)$ for $\mathrm{L}_{\mathrm{eq}}, \mathrm{L}_{10}$ and $\mathrm{L}_{50}$, while the contribution to $\mathrm{L}_{90}$ is roughly a factor of 2 .
The results are shown in Figure 3(b) where the measured noise level descriptors were plotted as functions of the logarithm of the traffic composition calculated with the best $w$. For $\mathrm{L}_{\text {eq }}, \mathrm{L}_{10}$ and $\mathrm{L}_{50}$, heavy vehicles (95\%) are the main source of noise. For $\mathrm{L}_{90}$, the contribution from light vehicles increases to about $10 \%$. When the standard deviation of the difference between the fitted and measured noise was taken, it was observed that all noise descriptors can be predicted with accuracies of around $\pm 2 \mathrm{~dB}(\mathrm{~A})$.

\section{CONCLUSION}

The study revealed that throughout the day, the noise generated by traffic is significantly high for all three types of roads categorized as A, B and C. Those who reside close to the traffic routes, although they are $30 \mathrm{~km}$ away from the Colombo city, are exposed to noise levels reaching $70 \mathrm{~dB}(\mathrm{~A})$ during day time for category $\mathrm{A}$ and $\mathrm{B}$ roads, which is the maximum recommended permissible noise level at roadsides for residential areas in Japan. This is aggravated by the fact that many of the schools, shops and commercial establishments are situated close to the traffic routes. Thus, noise regulatory mechanisms are required to lower the exposed noise levels to the acceptable standards.

The level of noise due to traffic varies with a number of parameters including the rate and the composition of vehicles. Specially, when the vehicle rate is high (more than 20 vehicles per minute), A-weighted equivalent noise levels $\mathrm{L}_{\text {eq }}$ exceeds $70 \mathrm{~dB}(\mathrm{~A})$. The investigation on the composition of vehicles on roads showed that heavy vehicles such as lorries, buses and containers contribute significantly to the average noise levels on main roads and secondary roads. For local roads, very light vehicles, such as motor bikes and three-wheelers contribute heavily to the average noise levels. The data also showed that for main roads, the average equivalent noise levels exceed $60 \mathrm{~dB}(\mathrm{~A})$ during night time. Thus, the present study recommends to carry out a community noise survey to implement medium- and long-term traffic noise reduction mechanisms.

A linear correlation was observed between $\mathrm{L}_{\text {eq }}$ and the other noise descriptors. It was shown that $\mathrm{L}_{10}$, which can be related to the annoyance, can be estimated with an accuracy of $\pm 1 \mathrm{~dB}(\mathrm{~A})$ by measuring $\mathrm{L}_{\text {eq }}$. It was also observed that the key noise descriptors can be estimated with an accuracy of $\pm 2.5 \mathrm{~dB}(\mathrm{~A})$ by measuring the rate of flow of vehicles. 


\section{Acknowledgement}

The authors are grateful to the Industrial Technology Institute, Colombo 07 for granting permission to use necessary instruments for sound measurements and supporting the field work.

\section{REFERENCES}

1. Ali S.A. \& Tamura A. (2003). Road traffic noise levels, restrictions and annoyance in Greater Cairo, Egypt. Applied Acoustics 64(8): $815-823$.

2. International Institute on Noise Control Engineering (I-INCE) (2009). Survey of legislations, regulations, and guidelines for control of community noise. Final Report of the I-Ince Technical Study Group on Noise Policies and Regulations (TSG 3). International Institute of Noise Control Engineering, USA.

3. Langdon F.J. (1976). Noise nuisance caused by road traffic in residential areas: part I. Journal of Sound and Vibration 47(2): $243-263$.

4. Manoel J., Filho A., Lenzi A., Henrique P. \& Zannin T. (2004). Effect of traffic composition on road noise: a case study. Transportation Research: part D 9(1): $75-80$. DOI: http://dx.doi.org/10.1016/j.trd.2003.08.001

5. Morillas J.M.B., Escobar V.G., Sierra J.A.M., Gomez R.V. \& Carmona J.T. (2002). An environmental noise study in the city of Caceres, Spain. Applied Acoustics 63(10): $1061-1070$.

6. Onuu M.U. (2000). Road traffic noise in Nigeria: measurement, analysis and evaluation of nuisance. Journal of Sound and Vibration 233(3): 391 - 405.

DOI: http://dx.doi.org/10.1006/jsvi.1999.2832

7. Ouis D. (2001). Annoyance from road traffic noise: a review. Journal of Environmental Psychology 21(1): 101 - 120. DOI: http://dx.doi.org/10.1006/jevp.2000.0187

8. Piccolo A., Plutino D. \& Cannistraro G. (2005). Evaluation and analysis of the environmental noise of Messina, Italy. Applied Acoustics 66(4): 447 - 465.

DOI: http://dx.doi.org/10.1016/j.apacoust.2004.07.005

9. Sommerhoff J., Recuero M. \& Suarez E. (2004). Community noise survey of the city of Valdivia, Chile. Applied Acoustics 65(7): $643-656$.

DOI: http://dx.doi.org/10.1016/j.apacoust.2004.01.003

10. Sooriyaarachchi R.T. \& Sonnadara D.U.J. (2008). Modelling free flowing vehicular traffic noise. Engineer 40(2): $43-47$.

11. Wewalwala S.N. \& Sonnadara D.U.J. (2011). Traffic noise enhancement due to speed bumps. Sri Lankan Journal of Physics 12: $1-6$.

12. World Health Organization (WHO) (1999). Adverse health effects of noise. Guidelines for Community Noise (eds. B. Berglund, T. Lindvall \& D. Schwela), pp. 39 - 54. World Health Organization, Geneva, Switzerland. 